Supporting Information

\title{
Single-component and mixed ferrocene-terminated alkyl monolayers covalently bound to $\mathrm{Si}(111)$ surfaces
}

\author{
Bruno FABRE* and Fanny HAUQUIER \\ Laboratoire d'Electrochimie Moléculaire et Macromoléculaire, Synthèse et Electrosynthèse \\ Organiques (UMR CNRS n 6510), Université de Rennes 1, Campus de Beaulieu, 35042 \\ Rennes Cedex, France. Fax: 3322323 6732; Tel: 3322323 6550; E-mail: fabre@univ- \\ rennes1.fr
}

Figure S1 XPS survey spectrum of a freshly prepared ferrocene-terminated monolayer covalently bound to a $\mathrm{Si}(111)$ surface. Takeoff angle: $45^{\circ}$.

Figure S2 XPS high-resolution spectra of the (a) C 1s, (b) Fe 2p and (c) Si 2p regions of a freshly prepared ferrocene-terminated monolayer (the $\mathrm{Si}-\mathrm{O}$ region is highlighted by the dashed circle).

Table S1 XPS high-resolution data obtained for a freshly prepared ferrocene-terminated monolayer. 


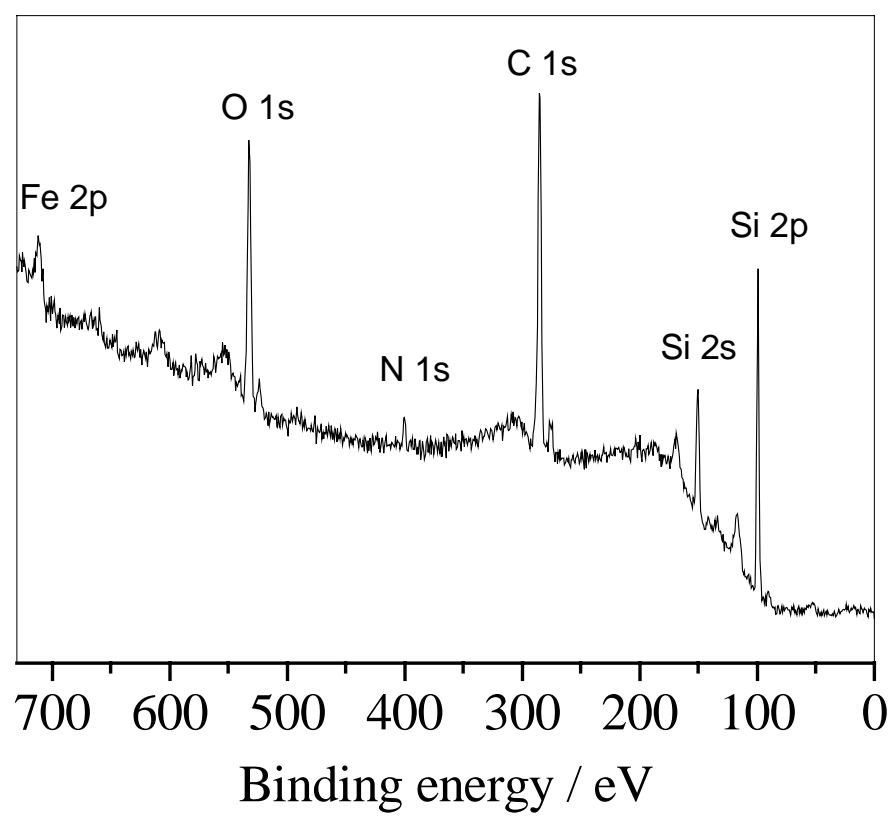

Figure S 1 

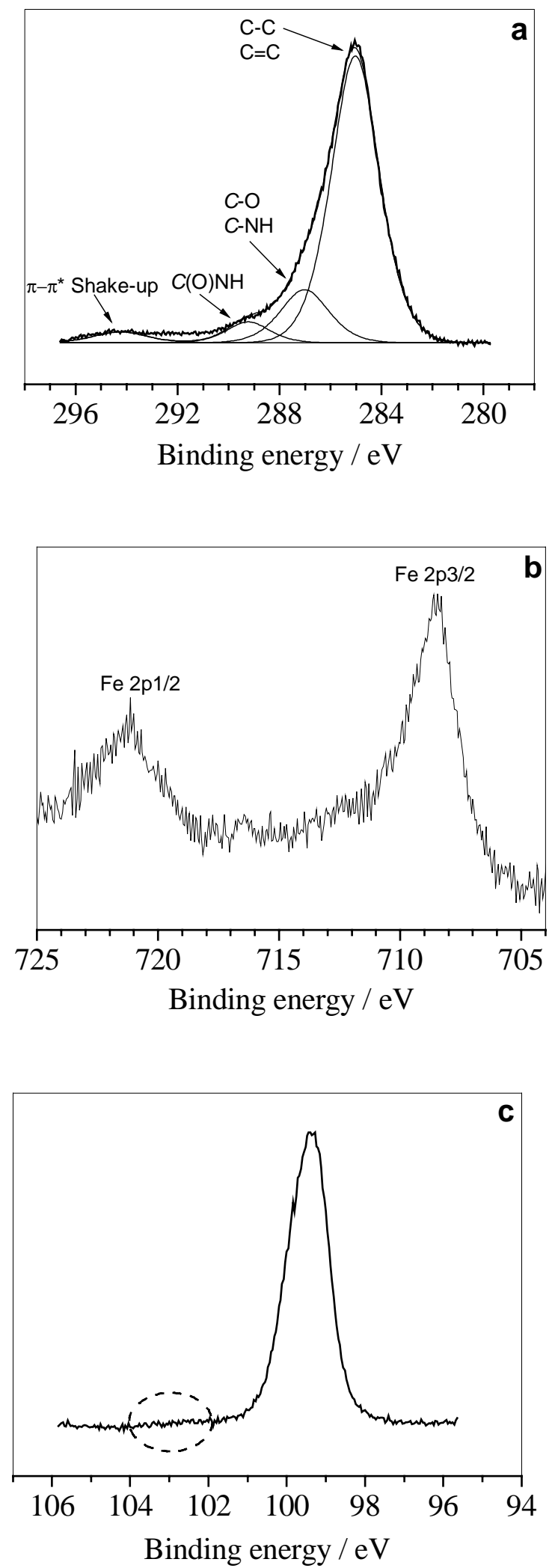

Figure S 2 


\begin{tabular}{|c|c|c|}
\hline Peak & Position / eV & Assignment \\
\hline \multirow[t]{3}{*}{$\mathrm{C} 1 \mathrm{~s}^{\mathrm{a}}$} & 285.0 & $C-C, C=C$ \\
\hline & 287.0 & $C-\mathrm{O}, C-\mathrm{N}$ \\
\hline & 289.2 & $C(\mathrm{O}) \mathrm{NH}$ \\
\hline \multirow[t]{3}{*}{$\mathrm{O} 1 \mathrm{~s}$} & 532.1 & $\mathrm{C}-O+$ adventitious oxygen- \\
\hline & & containingcontaminants \\
\hline & 533.7 & $\mathrm{C}=O$ \\
\hline $\mathrm{N} 1 \mathrm{~s}$ & 400.3 & $\mathrm{C}(\mathrm{O}) N \mathrm{H}$ \\
\hline \multirow[t]{2}{*}{$\mathrm{Fe} 2 \mathrm{p}$} & 708.4 & $\mathrm{Fe} 2 \mathrm{p}_{3 / 2}$ of ferrocene \\
\hline & 721.0 & Fe $2 p_{1 / 2}$ of ferrocene \\
\hline Si $2 p$ & 99.4 & $\mathrm{Si}-\mathrm{C}$ \\
\hline
\end{tabular}

${ }^{\text {a }}$ The shoulder at $294.2 \mathrm{eV}$ was attributed to a $\pi$ - $\pi^{*}$ shake-up satellite. ${ }^{1,2}$

\section{Table S1}

(1) Woodbridge, C. M.; Pugmire, D. L.; Johnson, R. C.; Boag, N. M.; Langell, M. A. J. Phys. Chem. B 2000, 104, 3085-3093.

(2) Mackie, N. M.; Castner, D. G.; Fisher, E. R. Langmuir 1998, 14, 1227-1235. 